\title{
Paramagnetic relaxation as seen by nuclear resonant forward scattering of synchrotron radiation
}

\author{
M. Haas and E. Realo \\ Institute of Physics, University of Tartu, EE-2400 Tartu, Estonia \\ H. Winkler, W. Meyer-Klaucke, ${ }^{*}$ and A. X. Trautwein \\ Institut für Physik, Medizinische Universität zu Lübeck, D-23538 Lübeck, Germany \\ O. Leupold ${ }^{\dagger}$ \\ II. Institut für Experimentalphysik, Universität Hamburg, D-22761 Hamburg, Germany
}

(Received 6 April 1999)

\begin{abstract}
Nuclear forward scattering of synchrotron radiation, which can be regarded as Mössbauer spectroscopy in the time domain, is a very promising tool for the investigation of dynamic phenomena. The interpretation of the resulting time-dependent intensity of the scattered radiation can be achieved by incorporating the stochastic theory of line shape into the program package SYNFOS, the theoretical basis of which has been described in an earlier paper [Haas et al., Phys. Rev. B 56, 14082 (1997)]. This is demonstrated by means of measurements carried out on the paramagnetic iron porphyrin complex $\left[\mathrm{Fe}\left(\mathrm{CH}_{3} \mathrm{COO}\right)\left(\mathrm{TP}_{\mathrm{piv}} \mathrm{P}\right)\right]^{-}$that exhibits dynamic effects due to spin-lattice relaxation at temperatures around $10 \mathrm{~K}$.
\end{abstract}

\section{INTRODUCTION}

In Ref. 1 formulas have been derived that describe nuclear resonant forward scattering of synchrotron radiation by randomly oriented ${ }^{57} \mathrm{Fe}$ complexes in the time domain. The amplitude of the coherent field of the scattered radiation has been described as the Fourier transform of the response function $\widetilde{R}_{\alpha \beta}$ of the system in the frequency domain that is determined by the forward-scattering amplitudes of the individual nuclei. A paramagnetic system is, e.g., an ironcontaining molecule with unpaired spins that couple to a total spin $S$. The $(2 S+1)$-fold paramagnetic degeneracy of the $\mathrm{Fe}$ ion can be lifted by the zero-field and Zeeman interactions ${ }^{1}$ [spin-Hamiltonian approximation, see also Eq. (8)]. Then an internal magnetic field is induced at the position of the nuclei caused by the spin $\vec{s}_{l}$, and the hyperfine coupling of the nuclear levels may be different in value and direction even for equivalent sites and for equivalent orientations of the paramagnetic complexes. (Here $\vec{s}_{l}$ $=\left\langle\phi_{l}^{\langle e l\rangle}|\hat{\vec{S}}| \phi_{l}^{\langle e l\rangle}\right\rangle$ is the expectation value of the electronic spin $\hat{\vec{S}}$ in the $l$ th spin subsystem.)

So far the coupling of the electronic spin of the Fe ion to the "heat-bath" type subsystems of the sample (phonons, spin subsystem of the sample, etc.) has been neglected in so much as we have assumed the paramagnetic relaxation, caused by such couplings, to be slow. In Ref. 1 the case of fast paramagnetic relaxation, i.e., random transitions between electronic spin substates, has been considered, too. In this approximation, the fast and frequent relaxation transitions are causing a stationary internal field that is determined by the thermal average of the spin vector

$$
\overrightarrow{\vec{s}}=\sum_{l} w_{l} \vec{s}_{l}
$$

where

$$
w_{l}=\exp \left[-\varepsilon_{l} /\left(k_{B} T\right)\right]\left\{\sum_{l^{\prime}} \exp \left[-\varepsilon_{l^{\prime}} /\left(k_{B} T\right)\right]\right\}^{-1},
$$

$\varepsilon_{l}$ is the energy of the $l$ th spin substate, $T$ is the temperature of the sample, and $k_{B}$ is the Boltzmann constant. In the intermediate relaxation cases (i.e., for moderate transition rates, comparable with the hyperfine splitting of the nuclear levels) such an approximation fails and the fluctuations of electronic spin $\vec{s}_{l}$ must be taken into account $\left[\vec{s}_{l} \rightarrow \vec{s}_{l}^{\mathrm{rel}}(t)\right]$. Hence, the formulas presented in Ref. 1 must be modified for applications that include time-dependent nuclear hyperfine Hamiltonians $\mathcal{H}^{(0)(e)}(t){ }^{2}$

\section{BASIC FORMULAS}

In the present contribution the paramagnetic relaxation is considered as a sequence of stochastic Markovian jumps of the electronic spin $\vec{S}$ between the spin substates, where $\vec{s}_{l}^{\text {rel }}(0)=\vec{s}_{l}$. Probabilities $w_{l l}$, of the transition jumps $l^{\prime}$ $\rightarrow l\left(\varepsilon_{l} \leqslant \varepsilon_{l^{\prime}}\right)$ satisfy the detailed balance condition:

$$
w_{l l^{\prime}}=\exp \left[\left(\varepsilon_{l^{\prime}}-\varepsilon_{l}\right) /\left(k_{B} T\right)\right] w_{l^{\prime} l} .
$$

In this approximation the evolution of the nuclear hyperfine states during the scattering processes is determined by the Liouville operator, 3,4

$$
\hat{L}=(1 / \hbar) \mathcal{H}_{n}^{x}+i \hat{W}
$$

instead of the exact time-dependent operator $\hat{L}(t)$ $=(1 / \hbar) \mathcal{H}_{n}^{x}(t)$. Here $\mathcal{H}_{n}^{x}$ is the superoperator, associated with the nuclear hyperfine Hamiltonians $\mathcal{H}^{(0)(e)}$ in the slow relaxation limit, i.e., its matrix elements are

$$
\begin{aligned}
\left\langle f^{\prime}, i^{\prime} ; l^{\prime}\left|\mathcal{H}_{n}^{x}\right| f, i ; l\right\rangle= & \left(\left\langle f^{\prime} ; l\left|\mathcal{H}^{(e)}\right| f ; l\right\rangle \delta_{i i^{\prime}}\right. \\
& \left.-\left\langle i ; l\left|\mathcal{H}^{(0)}\right| i^{\prime} ; l\right\rangle \delta_{f f^{\prime}}\right) \delta_{l l^{\prime}},
\end{aligned}
$$


where $f$ and $i$ indicate the hyperfine substates of the excited and ground nuclear level. $\hat{W}$ is the relaxation matrix with the elements $w_{l^{\prime} l} \delta_{i i^{\prime}} \delta_{f f^{\prime}}$ where the diagonal elements are determined as

$$
w_{l l}=-\sum_{l^{\prime}} w_{l^{\prime} l} .
$$

In such a stochastic model the resonant transition frequencies in the forward-scattering amplitudes are determined by the operator $\left[\left(\omega-\omega_{r}-i \Gamma / 2\right) \hat{E}-\hat{L}\right]^{-1}$, where $\omega_{r}$ is the transition frequency in the case of zero hyperfine splitting, $\Gamma$ is the transition width determined by the lifetime of the excited state of the nucleus, and $\hat{E}$ is the unit operator. After diagonalization of this operator the mean forward-scattering amplitudes $f_{\alpha \beta}(\omega)$ obtain the following form:

$$
\begin{aligned}
\bar{f}_{\alpha \beta}(\omega)= & \left(8 \pi^{2}\right)^{-1}(b / d) \int_{0}^{\pi} \sin \theta d \theta \int_{0}^{2 \pi} \int_{0}^{2 \pi} d \varphi d \psi \\
& \times \sum_{l} w_{l} \sum_{\mu, \mu^{\prime}= \pm 1,0}(-1)^{\mu} \\
& \times \Pi_{\alpha,-\mu}(\psi, \theta, \varphi) \Pi_{\beta, \mu^{\prime}}(\psi, \theta, \phi) \\
& \times \sum_{n} \bar{A}_{\mu \mu^{\prime}}^{(n)}(\theta, \varphi) /\left[\omega_{r}-\omega+\bar{\omega}_{n}(\theta, \varphi, l)-i \Gamma / 2\right],
\end{aligned}
$$

where $\psi, \theta, \varphi$ are the Euler angles, which specify the orientations of the complexes, and $\Pi_{\alpha \mu}(\psi, \theta, \varphi)$ are a combination of the Wigner functions $D_{\mu \mu^{\prime}}^{1}(\psi, \theta, \varphi)$, which couple the transition matrix elements in laboratory and molecular reference systems, ${ }^{5}$

$$
\Pi_{x \mu}=(1 / \sqrt{2})\left(D_{1 \mu}^{1}-D_{-1 \mu}^{1}\right)
$$

and

$$
\Pi_{y \mu}=(i / \sqrt{2})\left(D_{1 \mu}^{1}+D_{-1 \mu}^{1}\right) .
$$

$d$ is the geometric and $T_{M} \equiv 4 b$ the effective thickness of the absorber. In Eq. (5) the renormalized transition frequencies $\bar{\omega}_{n}(\theta, \varphi, l)$ are complex quantities,

$$
\bar{\omega}_{n}(\theta, \varphi ; l)=\Omega_{n}(\theta, \varphi ; l)-i \Gamma_{n}(\theta, \varphi ; l) / 2,
$$

where $n$ numerates the nuclear transitions in the complexes that have a fixed orientation. The number of these transitions is the same as in the case of slow relaxation, $\left(2 I_{0}+I\right)$ $\times\left(2 I_{e}+I\right) \times(2 S+I)$, where $I_{e}$ and $I_{0}$ are the values of the nuclear spin in the excited and ground states, but instead of the relative weights of the transitions we have complex quantities $\bar{A}_{\mu \mu^{\prime}}^{(n)}$ now.

When the relaxation transitions of the electronic spin $\vec{S}$ are uncorrelated in different ions the response function of the system $\widetilde{R}_{\alpha \beta}(\omega)$ can be expressed in terms of the forwardscattering amplitudes. The only difference here is that the forward-scattering amplitudes [Eq. (5)] are modified compared to Ref. 1. The complex shifts of the transition energies and of the corresponding frequencies (7) that are caused by the electron spin relaxation are different for the different transitions. Thus, the relaxation effects in the time-domain spectrum cannot be simply reduced to a single exponential factor, which would describe the general acceleration of the decay of the scattered pulse, but more subtle changes in the beat pattern have to be expected. In the range of moderate relaxation rates each transition is characterized by its corresponding line broadening $\Gamma_{n}$. As a result, both a faster decay of the pulse in total and a smearing of the beat pattern takes place. When the relaxation becomes faster $\left(w_{l^{\prime} l}>\left|\omega_{n}\right|\right)$ the situation changes. Now most of the transitions become very broad with small relative weights. On the other hand, we get $\Gamma_{n}(\theta, \varphi) \rightarrow 0$ for the intense transitions. Thus, a pronounced quantum-beat structure of the scattered radiation pulse appears again, although its shape is altered because a smaller number of transitions is associated with it. The number of such transitions is $\left(2 I_{e}+1\right)\left(2 I_{0}+1\right)$ for each orientation within paramagnetic complexes. The conditions of the fast relaxation limit as described above are fulfilled in this case and the corresponding formulas, which have already been given in Ref. 1, can be used in calculations.

Note that the fast decay of the pulses of coherently scattered radiation in the case of intermediate relaxation does not mean that decay of the excitation produced by the primary synchrotron radiation pulse in the sample is switched off. Due to incoherent processes (such as incoherent or multiphonon scattering or electron conversion) this decay goes on until the sample loses the stored energy. The nuclear lifetime $\Gamma^{-1}$ is the characteristic relaxation time for this process of energy relaxation. Simultaneously, however, an intensive dephasing phenomenon takes place in the sample caused by the paramagnetic relaxation of intermediate rate. A similar dephasing effect could be caused by the way also by a substantial variety of the nuclear transition frequencies. Thus the phase memory of the system can be destroyed in a time interval that is much shorter than $\Gamma^{-1}$. This phase relaxation of the system causes the decay of the coherent nuclear polarization as well as the decay of the strictly forward directed pulse of coherently scattered radiation coupled to such a polarization. Exactly the behavior of this forward-scattered pulse of radiation has been studied in this paper as well as in Ref. 1. The situation here is analogous to the paramagnetic resonance experiments where the stages of the transversal (loss of the phase memory) and the longitudinal (loss of the excess energy) relaxation can be distinguished in the relaxation process.

To calculate the forward-scattered synchrotron radiation pulses the program SYNFOS (Ref. 1) has been modified to enable its application to the systems where the intermediate paramagnetic relaxation takes place. The case of the interaction of the electronic spin with the acoustic phonons of long wavelength, conventionally denoted as direct process of spin-lattice relaxation, has been considered in the Debye continuum model. Here the one-phonon transition probabilities for $\varepsilon_{l} \leqslant \varepsilon_{l^{\prime}}$ have been expressed as follows:

$$
w_{l^{\prime} l}=w_{0} \frac{\left[\left(\varepsilon_{l^{\prime}}-\varepsilon_{l}\right) / k_{B}\right]^{p}}{\exp \left[\left(\varepsilon_{l^{\prime}}-\varepsilon_{l}\right) /\left(k_{B} T\right)\right]-1} .
$$

Two additional fitting parameters appear in Eq. (8): 


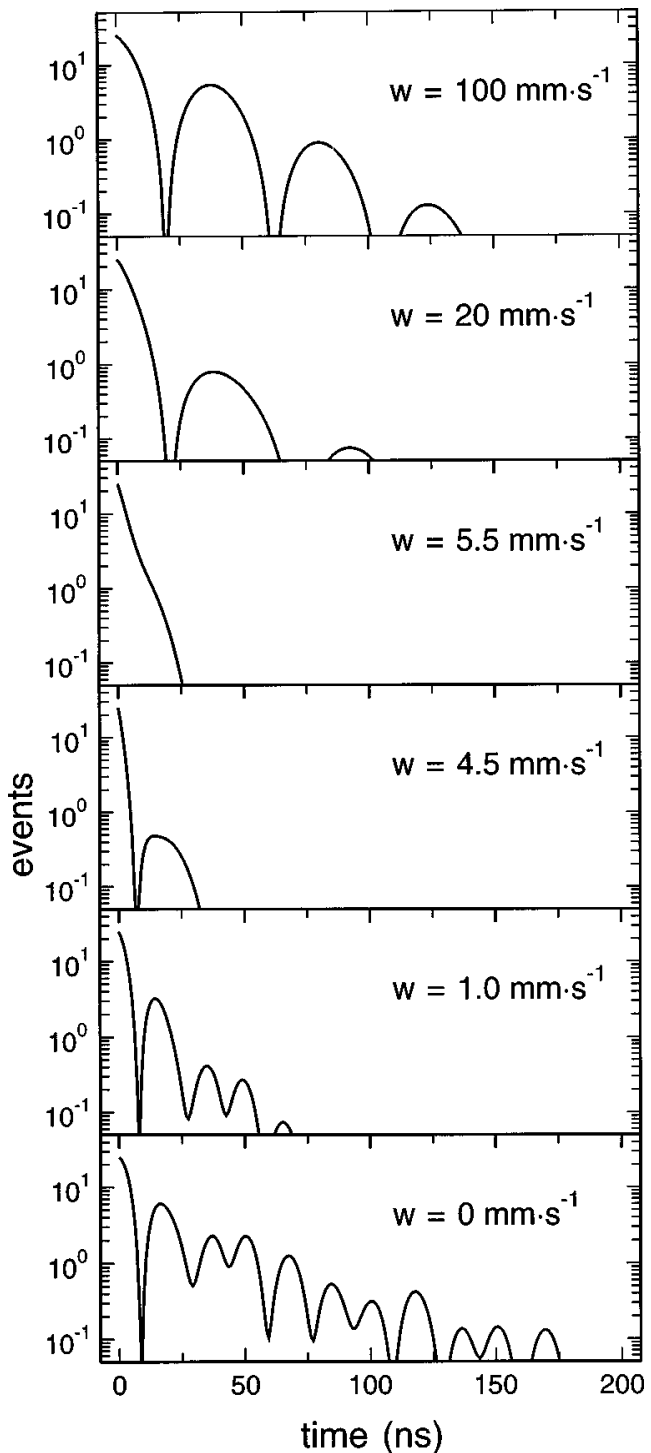

FIG. 1. Theoretical calculations of the nuclear forward scattering for the relaxation rates as indicated for a system with electron spin $S=\frac{1}{2}$, hyperfine parameters $A_{x, y, z} / g_{n} \beta_{n}=50 \mathrm{~T}$, and $\Delta E_{Q}$ $=2 \mathrm{~mm} \mathrm{~s}^{-1}$ in an external field of $75 \mathrm{mT}$ applied perpendicular to $\vec{k}$ and $\vec{\sigma}$.

(1) The constant $w_{0}$ that determines the strength of spinphonon coupling. It depends on the electronic transition matrix elements, phonon frequency distribution parameters, etc., ${ }^{4,5}$ but its dependence on the spin substate indices $l^{\prime}, l$ as well as on the temperature of the sample are neglected in our approximation.

(2) The power index $p$ that equals exactly to 3 in the Debye approximation. Its variation enables us to compensate to some extent the imperfection of the coarse continuum model. ${ }^{6}$

\section{COMPARISON WITH EXPERIMENTAL DATA}

In order to visualize the effects induced by paramagnetic relaxation, the time-dependent forward-scattering intensity has been calculated for a simple case and the resulting curves are presented in Fig. 1 for various relaxation rates. It is assumed that a polycrystalline ferric low-spin system, i.e.,

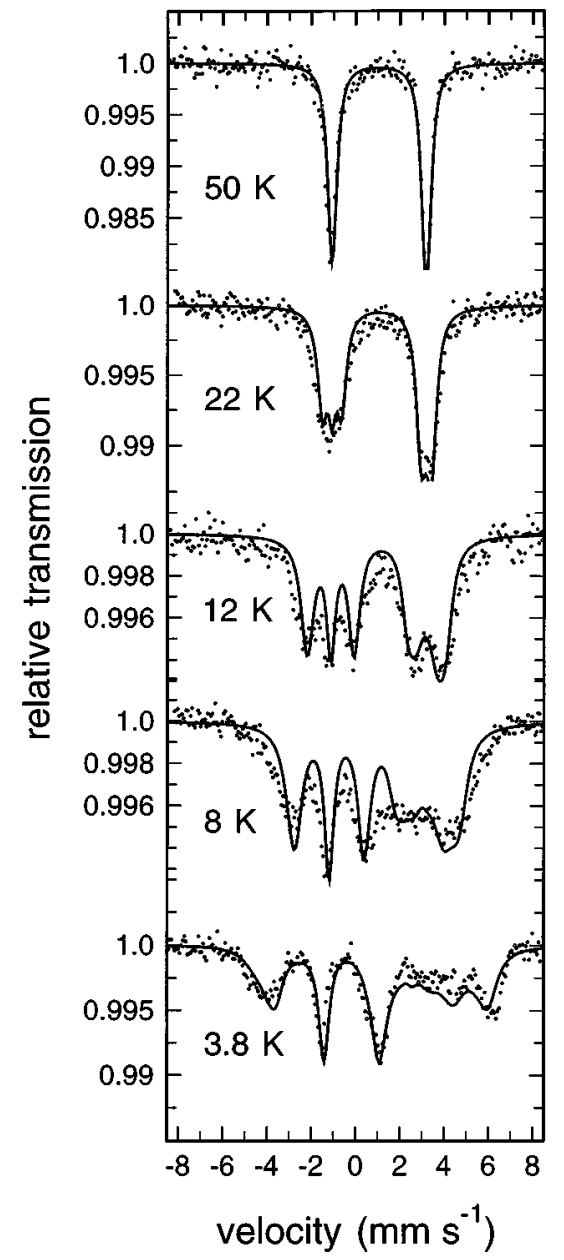

FIG. 2. Conventional Mossbauer spectra of complex $\left[\mathrm{Fe}\left(\mathrm{CH}_{3} \mathrm{COO}\right)\left(\mathrm{TP}_{\text {piv }} \mathrm{P}\right)\right]^{-}$taken at the temperatures as indicated in an external field of $4 \mathrm{~T}$ applied perpendicular to the $\gamma$ ray.

$S=\frac{1}{2}$, with effective thickness $T_{M}=20$ and values of $A_{x, y, z} / g_{n} \beta_{n}=-50 \mathrm{~T}$ and $\Delta E_{Q}=2 \mathrm{~mm} \mathrm{~s}^{-1}$ chosen for the hyperfine parameters is exposed to an external field $B_{\text {app }}$ $=75 \mathrm{mT}$ at a temperature of $100 \mathrm{~K}$. The transition rate from $l=-\frac{1}{2}$ to $l^{\prime}=+\frac{1}{2}$ is assumed to be given by Eq. (7) with $p=3$ and the rate for the reverse transition by Eq. (1).

With $B_{\mathrm{app}}=75 \mathrm{mT}$, it follows that $\left(\varepsilon_{l^{\prime}}-\varepsilon_{l}\right) / k_{B} \cong 0.1 \mathrm{~K}$ so that for $T=100 \mathrm{~K}$ one gets $w \equiv w_{l^{\prime} l}=w_{l l^{\prime}} \cong w_{0} \times 1 \mathrm{~K}^{3}$. Because the timescale for dephasing is determined by the hyperfine frequencies, it is reasonable to express the transition probabilities in units of $\mathrm{mm} \mathrm{s}^{-1}$ with $1 \mathrm{~mm} \mathrm{~s}^{-1}$ corresponding to $7.3 \times 10^{7} \mathrm{~s}^{-1}$. As the orientation of the applied field was chosen perpendicular to direction $\vec{k}$ and the polarization $\vec{\sigma}$ of the incoming beam only the two $\Delta m=0$ transitions are excited. Therefore at $w=0 \mathrm{~mm} \mathrm{~s}^{-1}$ essentially only one magnetic hyperfine frequency is observed, which is slightly modulated due to the powder average because this leads to different orientations of $V_{z z}$ with respect to the effective magnetic field at the position of the nucleus. At fast relaxation the effective magnetic field is only $75 \mathrm{mT}$ so that not more than the much slower electric quadrupole frequency is visible. Around $w=4.5 \mathrm{~mm} \mathrm{~s}^{-1}$, which corresponds approximately to the splitting between the two $\Delta m=0$ resonances, one can recognize the manifestation of the dephasing. From Fig. 1 it can also be verified that the curves start at $t=0 \mathrm{~ns}$ 


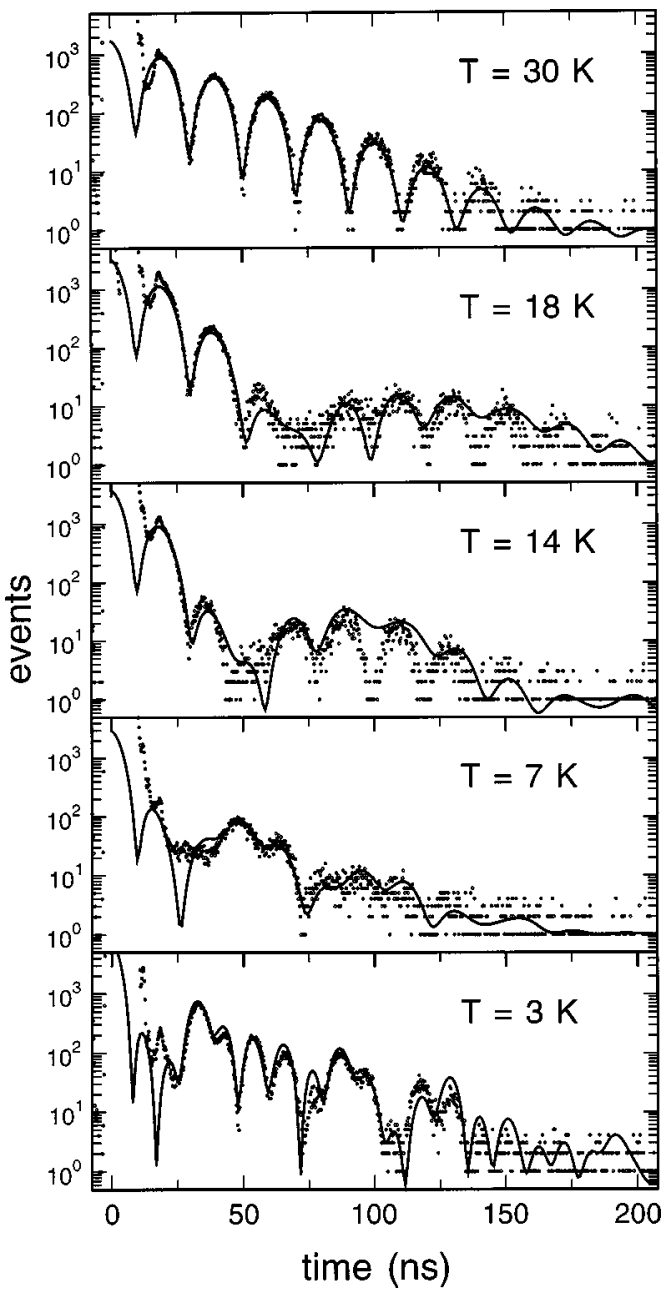

FIG. 3. Time dependence of the nuclear resonant forward scattering by the complex $\left[\mathrm{Fe}\left(\mathrm{CH}_{3} \mathrm{COO}\right)\left(\mathrm{TP}_{\text {piv }} \mathrm{P}\right)\right]^{-}$measured in an external field of $4 \mathrm{~T}$ applied perpendicular to $\vec{k}$ and $\vec{\sigma}$ at the temperatures as indicated.

with the value $b^{2}$, which is 25 for $T_{M}=20$, due to the normalization of the forward-scattering intensity that results from Eq. (5).

The so-called "picket-fence" iron porphyrin complex $\left[\mathrm{Fe}\left(\mathrm{CH}_{3} \mathrm{COO}\right)\left(\mathrm{TP}_{\text {piv }} \mathrm{P}\right)\right]^{-}$has been extensively studied by us with respect to its paramagnetic properties because it forms to some extent a model complex for the cytochrome P460 of the multiheme enzyme hydroxylamine oxidoreductase. ${ }^{7}$ It is a high-spin ferrous complex and its spin-Hamiltonian parameters have been determined in Ref. 1 at $3.3 \mathrm{~K}$ in an applied field of $6 \mathrm{~T}$ as $D=-0.8 \mathrm{~cm}^{-1}$ with $E / D=0$ for the zerofield splitting, $A_{x, y} / g_{n} \beta_{n}=-17 \mathrm{~T}$ and $A_{z} / g_{n} \beta_{n}=-12 \mathrm{~T}$ for the magnetic hyperfine-coupling parameters, $\Delta E_{Q}$ $=4.25 \mathrm{~mm} \mathrm{~s}^{-1}$ for the quadrupole splitting, and $\eta=0$ for the asymmetry parameter. Figure 2 shows the conventional Mössbauer spectra obtained at various temperatures in a field of $4 \mathrm{~T}$ applied perpendicular to the $\gamma$ ray. The spectra taken at 12 and at $22 \mathrm{~K}$ clearly exhibit the progressive collapse of the magnetic hyperfine splitting due to spin relaxation. Figure 3 gives the corresponding time-dependent nuclear resonant forward-scattering spectra taken with the setup available at beam line BW4 of HASYLAB in Hamburg from a sample of effective thickness $T_{M}=20$. The external field had again a

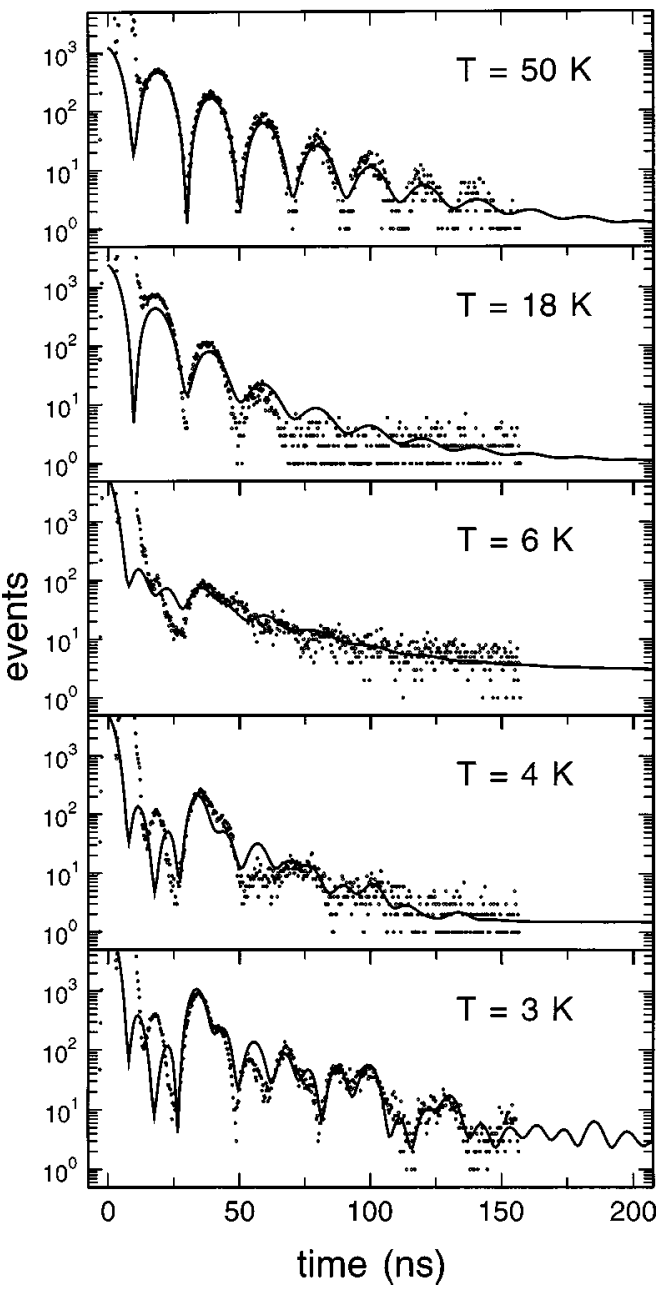

FIG. 4. Time dependence of the nuclear resonant forward scattering by the complex $\left[\mathrm{Fe}\left(\mathrm{CH}_{3} \mathrm{COO}\right)\left(\mathrm{TP}_{\text {piv }} \mathrm{P}\right)\right]^{-}$measured in an external field of $2 \mathrm{~T}$ applied perpendicular to $\vec{k}$ and $\vec{\sigma}$ at the temperatures as indicated.

strength of $4 \mathrm{~T}$ and was applied perpendicular to the wave vector $\vec{k}$ and to the polarization (electric-field vector) $\vec{\sigma}$ of the incoming beam.

The fits in Fig. 3 indicated by the full lines were performed for all temperatures by means of one single value of $3.65 \times 10^{5} \mathrm{~s}^{-1} \mathrm{~K}^{-3}$ for the scaling parameter $w_{0}$. The magnetic hyperfine-coupling tensor had to be readjusted slightly compared to our original analysis at $B_{\text {app }}=6 \mathrm{~T}$ and slow relaxation $^{1}$ giving $A_{x, y} / g_{n} \beta_{n}=-16.5 \mathrm{~T}$ and $A_{z} / g_{n} \beta_{n}=$ $-12 \mathrm{~T}$. In addition, the angular integration required for the powder samples used in the measurement showed that a certain degree of texture has to be taken into account. This was done by excluding $\theta \leqslant 40^{\circ}$ from the powder averaging in all fits, with $\theta$ denoting the angle between $\vec{B}_{\text {app }}$ and the molecular $z$ axis. The deviations between theory and experiment below $30 \mathrm{~ns}$ are due to the tail of the prompt peak and spurious bunches on top of it. In addition, it is striking that the experimental data taken at $14 \mathrm{~K}$ and at $18 \mathrm{~K}$ show more contrast in the beat structure than the theoretical spectra. This is probably due to effects that have been neglected up to now in the theory. The attenuation of the radiation pulse by electronic absorption when passing through the sample or the finite transversal and longitudinal coherence lengths might, 
e.g., limit the dephasing and suppress the manifestation of the smearing effect to some extent.

Measurements performed in an applied field of $2 \mathrm{~T}$ with the same orientation as for $4 \mathrm{~T}$ are presented in Fig. 4. The same value for $w_{0}$ applies, but again the magnetic hyperfinecoupling tensor had to be reduced a little by taking $A_{x, y} / g_{n} \beta_{n}=-15.5 \mathrm{~T}$ and $A_{z} / g_{n} \beta_{n}=-11$. The fit could be improved markedly by using an extended spin-Hamiltonian including cubic and axial fourth-order spin terms, ${ }^{8}$

$$
\begin{aligned}
\mathcal{H}_{s}= & D\left[S_{z}^{2}-\frac{1}{3} S(S+1)+\frac{E}{D}\left(S_{x}^{2}-S_{y}^{2}\right)\right] \\
& +\frac{1}{6} a\left[S_{x}^{4}+S_{y}^{4}+S_{z}^{4}-\frac{1}{5} S(S+1)\left(3 S^{2}+3 S-1\right)\right] \\
& +\frac{1}{180} F\left[35 S_{z}^{4}-30 S(S+1) S_{z}^{2}+25 S_{z}^{2}-6 S(S+1)\right. \\
& \left.+3 S^{2}(S+1)^{2}\right]+\beta \vec{S}_{g} \vec{B}
\end{aligned}
$$

with $a=1.2 \mathrm{~cm}^{-1}$ and $F=3.0 \mathrm{~cm}^{-1}$. Obviously, the effect of these fourth-order terms had been insignificant before in the presence of the strong Zeeman interaction.

\section{SUMMARY}

Relaxation as seen by nuclear resonant forward scattering can be treated theoretically on the same basis as described in Ref. 1 only that the transition frequencies and amplitudes become complex quantities as they result from the diagonalization of the corresponding Liouville operator, the form of which is well known in conventional Mössbauer spectroscopy from the stochastic theory of line shape. Data taken from the iron porphyrin complex $\left[\mathrm{Fe}\left(\mathrm{CH}_{3} \mathrm{COO}\right)\left(\mathrm{TP}_{\text {piv }} \mathrm{P}\right)\right]^{-}$ in external fields of 2 and $4 \mathrm{~T}$ have been successfully interpreted in the framework of this theory and the presence of the direct process of spin-lattice relaxation in the temperature range between 3 and $30 \mathrm{~K}$ has been proven. This example demonstrates that the interpretation of experimental data from systems that exhibit time-dependent hyperfine interactions is possible in a satisfactory manner by incorporation of the stochastic theory into the SYNFOS program package.

The advantages of the application of nuclear forward scattering for the study of dynamical phenomena as compared to Mössbauer absorption spectroscopy are expected to result from its sensitivity to phase shifts of the beats, because in the time-domain amplitudes are added and not intensities as in the energy domain, and from the exploitation of polarization effects. However, to demonstrate these advantages in detail we will need further studies. Thus in the present work, it was predominantly the technical aspects such as the small size of the sample used and the reduction in measuring time that have made the method attractive.

\section{ACKNOWLEDGMENT}

This work was supported by the BMBF Grants No. 05643 FLA 3 and No. 05 SK8 FLA 8.
*Present address: EMBL-Outstation Hamburg, D-22603 Hamburg, Germany.

†Present address: ESRF, Nuclear Resonance Group, F-38043 Grenoble Cedex, France.

${ }^{1}$ M. Haas, E. Realo, H. Winkler, W. Meyer-Klaucke, A. X. Trautwein, and O. Leupold, Phys. Rev. B 56, 14082 (1997).

${ }^{2}$ A. X. Trautwein, E. Bill, E. L. Bominaar, and H. Winkler, Struct. Bonding (Berlin) 8, 1 (1991).

${ }^{3}$ R. A. Edmonds, Angular Momentum in Quantum Mechanics (Princeton University Press, Princeton, NJ, 1957).

${ }^{4}$ H. Winkler, C. Schulz, and P. G. Debrunner, Phys. Lett. 69A, 360
(1979).

${ }^{5}$ H. Winkler, E. Bill, A. X. Trautwein, A. Kostikas, A. Simopoulos, and A. Terzis, J. Chem. Phys. 89, 732 (1988).

${ }^{6}$ H. Winkler, X.-Q. Ding, M. Burghardt, A. X. Trautwein, and F. Parak, Hyperfine Interact. 91, 875 (1994).

${ }^{7}$ E. L. Bominaar, X.-Q. Ding, A. Gismelseed, E. Bill, H. Winkler, A. X. Trautwein, H. Nasri, J. Fischer, and R. Weiss, Inorg. Chem. 31, 1845 (1992).

${ }^{8}$ A. Abragam and M. Bleaney, Electron Paramagnetic Resonance of Transition Ions (Clarendon, Oxford, 1970), p. 437. 